\title{
Coupling FEM, Bloch Waves and TMM in meta poroelastic laminates
}

\author{
M. Gaborit ${ }^{1,3)}$, L. Schwan ${ }^{1)}$, O. Dazel ${ }^{1)}$, J.-P. Groby ${ }^{1)}$, \\ T. WeISSER ${ }^{2)}$, P. GÖRANSSON ${ }^{3)}$ \\ 1) LAUM, UMR CNRS 6613, Université du Maine, Le Mans, France \\ 2) MIPS Laboratory, Université de Haute-Alsace, Mulhouse, France \\ 3) MWL, KTH Royal Institute of Technology, Stockholm, Sweden \\ Contact: gaborit@kth.se
}

\section{Summary}

The propagation of airborne plane waves in the presence of a meta poroelastic laminate, that is a poroelastic matrix coated with thin elastic layers at its facings 5 and periodically-embedded with inclusions, is studied. Using the Finite Element Method (FEM) only would result in a drastic increase of the degrees of freedom due to the fine mesh required to account for the very thin coatings. Here, the approach relies on: the Bloch 10 wave expansion of the fields in air; the modal Transfer Matrix Method to account for the coatings; and the coupling with the FEM model of the poroelastic matrix and the resonant inclusions. The model is developed for reflection and transmission problems 15 and it can account for coatings with multiple layers. The procedure induces the addition of the Bloch coefficients in the FEM's linear system at a negligible additional computational cost. It is applied to the meta poroelastic laminates with poroelastic inclusions

20 and rubber shell inclusions. The results are compared with those from the Multiple Scattering Theory and an excellent agreement between the methods is found. The approach offers a numerically-efficient way to account for coatings applied to meta poroelastic layers,

25 and finds applications in industrial prototypes where coatings are widely used.

\section{Introduction}

Sound insulation is a prominent research topic in acoustics and the development of tailored absorbers using structured media has gained even more interest with the emergence of the so-called metamaterials $[1-5]$. Recently, the use of meta-poroelastic media, consisting of micro-structures periodically embedded in a poroelastic matrix, has been put for-

35 ward $[4,6,7]$. Such materials rely on the acoustic/elastodynamic energy reduction, while energy dissipation is achieved through visco-thermal phenomena in the matrix pores.

Besides, audacious designs are becoming more and 40 more accessible, and new manufacturing solutions such as 3-D printing [8-10] now allow materializing algorithmically-generated structures with a high fidelity. In particular, manufacturing numerous quasiidentical unit cells is made possible and allows for full scale measurements. Such prototyping techniques motivate the use of advanced optimization techniques such as genetic algorithms or nonlinear programming to simulate systems under realistic conditions $[11,12]$.

To this end, efficient and accurate numerical models of systems are required and several options have been considered. On one hand, semi-analytical approaches such as Multiple Scattering Theory [13], allow for very fast evaluation of system responses at the cost of an initial analytical resolution. Nevertheless, Multiple Scattering Theory (MST) is limited to simple inclusion geometries. On the other hand, more versatile purely numerical techniques may be used to model one elementary cell with periodicity conditions but tend to exhibit a much higher computational cost at each evaluation. The Finite-Element Method (FEM) is part of this second group and the cost of evaluation is directly linked to the mesh refinement which, in turn, is related to both the frequency of interest and the size of the smallest geometrical features. The latter is of particular interest in the scope of metaporoelastic systems for which the free surface may be protected by thin layers (e.g. films or fabric) that have an impact on the acoustic response and tend to drive the mesh refinement, increasing considerably the computational cost. Considering these points, it proves interesting to develop a method combining the versatility and ease of modelling associated to the FEM while alleviating the cost of added layers by accounting for them separately.

The present work is concerned with the plane wave reflection/transmission of airborne sound from a laminate panel made of a metaporoelastic layer sandwiched between very thin elastic coatings. A method is presented which couples the FE model of a metaporoelastic layer and the Bloch expansions of the fields in the surrounding media. The proposed approach efficiently accounts for elastic coatings that may be added to the poroelastic matrix and modelled with only a few additional degrees of freedom. The 
85 model can give access to the reflection and transmission coefficients not only for the specular modes but also for the Bloch modes of higher order. The problem is tackled in 2-D and no particular assumption is made about the inner design of the metaporoelas-

90 tic layer. The method is presented in Section 2, and the results are compared with those from the Multiple Scattering Theory (MST) for poroelastic media [6] in Section 3.

\section{Numerical model}

\subsection{Statement of the problem}

The meta-poroelastic laminate consists of the infinite $D$-periodic arrangement along the $x$ axis of the elementary cell made of (1) a poroelastic matrix; (2) one or several periodically embedded inclusions of arbitrary shape in the poroelastic matrix; and (3) a thin elastic coating at the plane boundary $\Gamma_{-}$of the poroelastic matrix, see figure 1 . The coating is supposed to be made of homogeneous elastic material. Furthermore, the exact nature of the inclusions is not specified at this stage, but they are supposed to be sufficiently long to tackle the problem in the 2-D cross section $(x, z)$. In this Cartesian coordinate system, the interface $\Gamma_{+}$is given by $z=z_{+}$while the coating boundary $\Gamma_{-}$on the other side is given by $z=z_{-}$.

110 For simplicity in the derivation, the meta-poroelastic laminate is arranged against a rigid backing at the boundary $\Gamma_{b}$ and a single-layer coating is considered. The model will be extended to transmission problems and multi-coating configurations in Section 2.4.

115 The meta-poroelastic laminate is supposed to be in contact with air at the boundary $\Gamma_{-}$, see sigure 1 . The reflection of an airborne unitary plane wave from the meta-poroelastic laminate is studied in the linear harmonic regime at the circular frequency $\omega$ (using $e^{j \omega t}$

120 time convention). Taking advantage of the adaptivity of the FEM and its ability to model various types of inclusions, the meta-poroelastic layer comprising the poroelastic matrix with the inclusion will be modelled using the FEM. As a first step to the Finite Element 125 Method [14], the equations governing the equilibrium in the poroelastic matrix and the inclusions are formulated in their weak form. The two classical approaches to model poroelastic with the FEM use the solid displacement $\mathbf{u}^{s}$ and interstitial pressure $p$ as 30 main variables. They differ in their way of accounting for boundaries: Ref. [15] proposes a boundary operator coupling naturally with fluid media, whereas the formulation in Ref. [16] couples best with elastic and poroelastic media. This last formulation uses the total stress tensor $\sigma^{t}$ and relative displacement $\mathbf{w}$ between solid and fluid phase as secondary variables used in the boundary operators. While the full weak form associated to the FEM domain is beyond the scope of the present work, the expression of the boundary

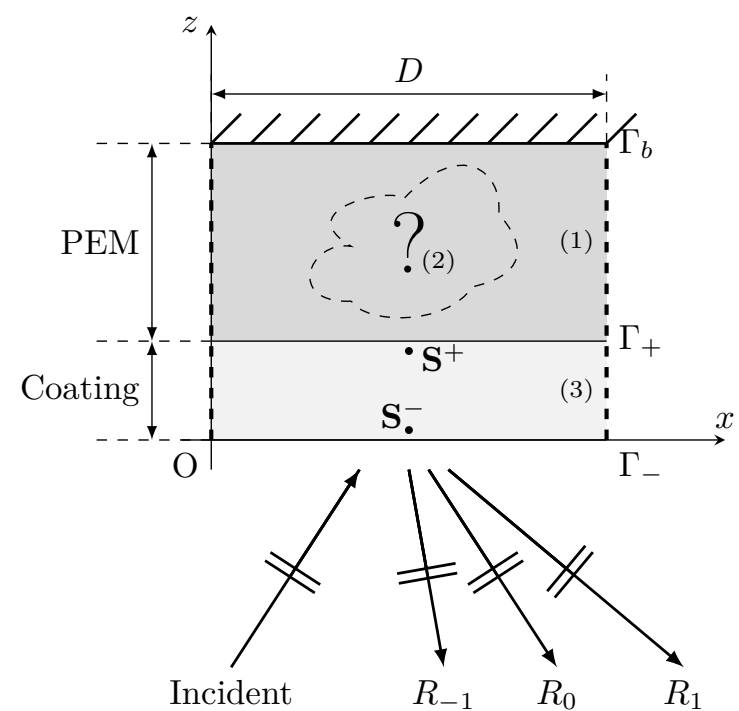

Figure 1: An generic elementary cell of an infinitely periodic system. The dotted lines on each side denotes periodicity conditions (applied in the FE scheme). A detailed description of the cell is given in section 2 . The inclusion is not detailed and is of very little importance: only counts the homogeneity of the interface $\Gamma_{+}$.

operator along $\Gamma_{+}$, here expressed with the normal $\mathbf{n}$ pointing outwards the FE domain, is given by:

$$
\mathcal{I}=\int_{\Gamma_{+}} \delta \mathbf{u}^{s} \cdot\left[\underline{\underline{\sigma}}^{t} \cdot \mathbf{n}\right] \mathrm{d} \Gamma+\int_{\Gamma_{+}} \delta p[\mathbf{w} \cdot \mathbf{n}] \mathrm{d} \Gamma .
$$

A crucial point is that all the other boundaries of the FE domain are treated classically by the FEM. They will not be discussed in the present paper.

Evaluating this integral is hence one key of the FEM model. To avoid modeling the coating with the FEM (which would inevitably lead to a drastic increase in the number of degrees of freedom due to the very fine mesh required by very small thickness), an alternative procedure is presented here, that takes advantage of the quasi-periodicity in the system. The present approach relies on the transfer of the unknown Bloch coefficients from the fluid medium to the poroelastic matrix. A technique similar to the transfer matrix method is used for transferring the Bloch expansions and allows to account for the coating by rewriting the FEM boundary operator $\mathcal{I}$ and the associated set of continuity conditions.

\subsection{Bloch wave expansions and modal Transfer Matrix Method}

Due to the $D$-periodicity of the meta-poroelastic laminate, the pressure field $p^{a}$ in air can be expanded as follows in terms of Bloch waves: 


$$
p^{a}(\mathbf{r})=\sum_{l \in \mathbb{Z}}\left[\delta_{0 l} e^{-j k_{z}^{l} z}+R_{l} e^{+j k_{z}^{l} z}\right] e^{-j k_{x}^{l} x}
$$

where $\delta_{0 l}$ is the Kronecker symbol and represents

the amplitude of the incident wave, $R_{l}$ are complex reflection coefficients and $k_{x}^{l}$ and $k_{z}^{l}$ are the Bloch wavenumbers defined as:

$$
k_{x}^{l}=k_{x}^{i}+\frac{2 \pi l}{D} ; \quad k_{z}^{l}=\sqrt{\left(\frac{\omega}{c}\right)^{2}-\left(k_{x}^{l}\right)^{2}},
$$

where $k_{x}^{i}$ is the wavenumber of the incident field in the direction $x, c$ is the sound speed in the fluid medium of density $\rho$. Besides, the particle displacement $\mathbf{u}^{a}=\left\{u_{x}^{a}, u_{z}^{a}\right\}^{T}$ in air is given by the momentum conservation $\rho \omega^{2} \mathbf{u}^{a}=\operatorname{grad}\left(p^{a}\right)$ and, using equation (2), its component along $z$ reads:

$$
u_{z}^{a}=\sum_{l \in \mathbb{Z}}\left[u_{z}^{a, i} \delta_{0 l} e^{-j k_{z}^{l} z}+u_{z}^{a, l} R_{l} e^{+j k_{z}^{l} z}\right] e^{-j k_{x}^{l} x},
$$

where the harmonic displacement coefficients $u_{z}^{a, i}$ for the incident field and $u_{z}^{a, l}$ for the Bloch reflections read:

$$
u_{z}^{a, i}=\frac{-j k_{z}^{i}}{\rho \omega^{2}} ; \quad u_{z}^{a, l}=\frac{j k_{z}^{l}}{\rho \omega^{2}} .
$$

Similarly, the solid particle displacement $\mathbf{u}^{e}\left(x, z_{ \pm}\right)$ at the boundary $\Gamma_{ \pm}$of the elastic coating (fixed value of $z=z_{ \pm}$) can be expanded as:

$$
\mathbf{u}^{e}\left(x, z_{ \pm}\right)=\sum_{l \in \mathbb{Z}}\left[\mathbf{u}^{e, l}\left(z_{ \pm}\right)\right] e^{-j k_{x}^{l} x},
$$

where the vectors $\mathbf{u}^{e, l}\left(z_{ \pm}\right)=\left\{u_{x}^{e, l}\left(z_{ \pm}\right), u_{z}^{e, l}\left(z_{ \pm}\right)\right\}^{T}$ are the complex amplitudes of the displacement $\mathbf{u}^{e}\left(x, z_{ \pm}\right)$in its Spatial Fourier Transform along $x$, that is its projection on Bloch waves. Likewise, the surface stress vector $\boldsymbol{\sigma}^{e}=\left\{\sigma_{z x}^{e}, \sigma_{z z}^{e}\right\}^{T}$ at the boundary $\Gamma_{ \pm}$is expanded as:

$$
\boldsymbol{\sigma}^{e}\left(x, z_{ \pm}\right)=\sum_{l \in \mathbb{Z}}\left[\boldsymbol{\sigma}^{e, l}\left(z_{ \pm}\right)\right] e^{-j k_{x}^{l} x} .
$$

At the interface $\Gamma_{-}$between the fluid medium and the coating, that is at $z=z_{-}$, the following boundary conditions hold, stating the continuity of the normal flux and of the surface stress vector:

$$
u_{z}^{e}=u_{z}^{a} ; \quad \sigma_{z x}^{e}=0 ; \quad \sigma_{z z}^{e}=-p^{a} \quad \text { at } \Gamma_{-} .
$$

Note that the $x$-component of the displacement $\mathbf{u}^{e}$ does not appear in those conditions, and its modal components $u_{x}^{e, i}\left(z_{-}\right)$and $u_{x}^{e, l}\left(z_{-}\right)$are hence unknowns of the problem. Using equations (2), (4), (6), (7) and the orthogonality of the Bloch waves, the interface conditions (8) leads to the following relations, where the Bloch modes are decoupled from one another:

$$
\left\{\begin{array}{l}
u_{x}^{e, l}\left(z_{-}\right)=\text {unknown, } \\
u_{z}^{e, l}\left(z_{-}\right)=u_{z}^{a, i} \delta_{0 l}+u_{z}^{a, l} R_{l}, \\
\sigma_{z x}^{e, l}\left(z_{-}\right)=0, \\
\sigma_{z z}^{e, l}\left(z_{-}\right)=-\delta_{0 l}-R_{l} .
\end{array}\right.
$$

The components on the left-hand side of equations (9) can be advantageously arranged in the state vector $\mathbf{S}_{l}^{-}$for Bloch mode $l$ at the boundary $\Gamma_{-}\left(z=z_{-}\right)$as follows:

$$
\mathbf{S}_{l}^{-}=\left\{u_{x}^{e, l}\left(z_{-}\right), u_{z}^{e, l}\left(z_{-}\right), \sigma_{z x}^{e, l}\left(z_{-}\right), \sigma_{z z}^{e, l}\left(z_{-}\right)\right\}^{T} .
$$

Then, using expression (5) for the displacements $u_{z}^{a, i}$ and $u_{z}^{a, l}$, equation (9) can be written in the following matrix formulation:

$$
\mathbf{S}_{l}^{-}=\mathbf{E}^{-} \delta_{0 l}+\left[\mathbf{I}_{l}^{-}\right] \mathbf{Q}_{l},
$$

with the vector $\mathbf{E}^{-}$being related to the incident field, $\mathbf{Q}_{l}=\left\{u_{x}^{e, l}\left(z_{-}\right), R_{l}\right\}^{T}$ being the vector of unknowns and $\left[\mathbf{I}_{l}^{-}\right]$a matrix implementing the continuity conditions for the reflections. These quantities are defined by:

$$
\mathbf{E}^{-}=\left\{\begin{array}{c}
0 \\
\frac{-j k_{z}^{i}}{\rho \omega^{2}} \\
0 \\
-1
\end{array}\right\} ; \quad\left[\mathbf{I}_{l}^{-}\right]=\left[\begin{array}{cc}
1 & 0 \\
0 & \frac{j k k_{z}^{l}}{\rho \omega^{2}} \\
0 & 0 \\
0 & -1
\end{array}\right]
$$

Likewise, the state vector $\mathbf{S}_{l}^{+}$for the Bloch mode $l$ at the boundary $\Gamma_{+}\left(z=z_{+}\right)$reads:

$$
\mathbf{S}_{l}^{+}=\left\{u_{x}^{e, l}\left(z_{+}\right), u_{z}^{e, l}\left(z_{+}\right), \sigma_{z x}^{e, l}\left(z_{+}\right), \sigma_{z z}^{e, l}\left(z_{+}\right)\right\}^{T} .
$$

Considering the orthogonality of the Bloch waves (exponential functions), each Bloch mode $l$ at the boundary $\Gamma_{+}$depends linearly on the Bloch mode of the same order $l$ at the boundary $\Gamma_{-}$. Introducing a modal transfer matrix $\left[\mathbf{T}_{l}\right]$ for each Bloch mode $l$, this linear relation is given by the relation:

$$
\mathbf{S}_{l}^{+}=\left[\mathbf{T}_{l}\right] \mathbf{S}_{l}^{-} .
$$

The expression for the modal transfer matrices $\left[\mathbf{T}_{l}\right]$ related to the elastic coating can be found in Ref. [17]. Now, substituting (11) into (14), the state vector $\mathbf{S}_{l}^{+}$ is found to take the form:

$$
\mathbf{S}_{l}^{+}=\mathbf{E}^{+} \delta_{0 l}+\left[\mathbf{I}_{l}^{+}\right] \mathbf{Q}_{l},
$$

where the vector $\mathbf{E}^{+}$and the matrix $\left[\mathbf{I}_{l}^{+}\right]$are given by:

$$
\mathbf{E}^{+}=\left[\mathbf{T}_{0}\right] \mathbf{E}^{-} ; \quad\left[\mathbf{I}_{l}^{+}\right]=\left[\mathbf{T}_{l}\right]\left[\mathbf{I}_{l}^{-}\right] .
$$

Note in equation (15) that the state vector $\mathbf{S}_{l}^{+}$for the Bloch mode $l$ at the boundary $\Gamma_{+}$actually depends on the vector $\mathbf{Q}_{l}$ of unknowns, that are the 


\subsection{FEM implementation}

At the interface $\Gamma_{+}$between the elastic coating and the poroelastic matrix, the following conditions hold, the continuity of the normal surface stress and solid displacements:

$$
\mathbf{w} \cdot \mathbf{n}=0 ; \quad \underline{\underline{\sigma}}^{t} \cdot \mathbf{n}=-\boldsymbol{\sigma}^{e} ; \quad \mathbf{u}^{s}=\mathbf{u}^{e} \quad \text { at } \Gamma_{+} \cdot
$$

The minus sign in the balance of surface stresses has been introduced to comply with the orientation of the normal vector at $\Gamma_{+}$. Using equations (7) and (13), the surface stress $\underline{\underline{\sigma}}^{t} \cdot \mathbf{n}=-\boldsymbol{\sigma}^{e}\left(z_{+}\right)$at $\Gamma_{+}$reads:

$$
\underline{\underline{\sigma}}^{t} \cdot \mathbf{n}\left(x, z_{+}\right)=-\left.\sum_{l \in \mathbb{Z}} \mathbf{S}_{l}^{+}\right|_{3,4} e^{-j k_{x}^{l} x}
$$

where the subscripts 3,4 denote the extraction of the 3rd and 4th row of the tensor. Combining equation (18) with (15) results in:

$$
\underline{\underline{\sigma}}^{t} \cdot \mathbf{n}\left(x, z_{+}\right)=-\sum_{l \in \mathbb{Z}}\left(\left.\mathbf{E}^{+}\right|_{3,4} \delta_{0 l}+\left.\left[\mathbf{I}_{l}^{+}\right]\right|_{3,4} \mathbf{Q}_{l}\right) e^{-j k_{x}^{l} x} .
$$

Substituting this expression back into the expression of $\mathcal{I}$ in equation (1) and using the impervious surface condition $\mathbf{w} \cdot \mathbf{n}=0$ at $\Gamma_{+}$from equation (18), the boundary operator reads:

$$
\begin{aligned}
\mathcal{I}= & -\int_{\Gamma_{+}}\left(\left.\mathbf{E}^{+}\right|_{3,4} e^{-j k_{x}^{l} x}\right) \cdot \delta \mathbf{u}^{s} \mathrm{~d} \Gamma \\
& -\sum_{l \in \mathbb{Z}} \int_{\Gamma_{+}}\left(\left.\left[\mathbf{I}_{l}^{+}\right]\right|_{3,4} \mathbf{Q}_{l} e^{-j k_{x}^{l} x}\right) \cdot \delta \mathbf{u}^{s} \mathrm{~d} \Gamma
\end{aligned}
$$

Similarly, using the last condition of (17) as well as equations (13) and (6), the displacement at the surface of the poroelastic matrix is retrieved. Directly substituting $\mathbf{S}_{l}^{+}$from (15), $\mathbf{u}^{s}$ reads:

$$
\mathbf{u}^{s}=-\sum_{l \in \mathbb{Z}}\left(\left.\mathbf{E}^{+}\right|_{1,2} \delta_{0 l}+\left.\left[\mathbf{I}_{l}^{+}\right]\right|_{1,2} \mathbf{Q}_{l}\right) e^{-j k_{x}^{l} x}
$$

where the subscripts 1,2 denote the extraction of the first and second rows of the vector or matrix.

Projecting the continuity of the solid displacement in equation (21) on each Bloch mode along $\Gamma_{+}$and usunknown coefficients and the fields from the FEM:

$\int_{0}^{D} \mathbf{u}^{s}\left(x, z_{+}\right) e^{j k_{x}^{l} x} \mathrm{~d} x=\left.D \mathbf{E}^{+}\right|_{1,2} \delta_{0 l}+\left.D\left[\mathbf{I}_{l}^{+}\right]\right|_{1,2} \mathbf{Q}_{l}$

Both equations (20) and (22) can now be implemented in the FEM model of the poroelastic matrix with the inclusion. After the spacial discretization and the Galerkin expansion over a set of shape functions, the FEM process results in a linear system:

$$
[\mathbf{A}] \mathbf{X}=\mathbf{B},
$$

where the matrix $[\mathbf{A}]$ describes the propagation in the volume; the vector $\mathbf{B}$ comes from the discretization of the boundary operator; and the vector $\mathbf{X}$ contains the $N_{F E}$ nodal values for the fields [14].

To perfectly represent the reflected field, the Bloch wave expansion involves infinite sums on $\mathbb{Z}$, but for practical reasons, only a truncation to $2 N+1$ terms ranging from $-N$ to $N$ is considered. The integer $N$ is chosen according to an empirical rule based on previous numerical experiments $[6,18,19]$ and is large enough for the Bloch waves' series to represent the fields' complexity:

$$
N=\left\lfloor\frac{D}{2 \pi}\left(3 \operatorname{Re}\left(\frac{\omega}{c}\right)-k_{x}^{0}\right)\right\rfloor+5,
$$

where $\operatorname{Re}(x)$ represents the real part of $x$ and 5 is chosen as a security term.

The vectors $\mathbf{Q}_{l}$ of unknowns are gathered in the overall vector $\mathbf{Q}$ :

$$
\mathbf{Q}=\left\{\mathbf{Q}_{-N} \ldots \mathbf{Q}_{-1}, \mathbf{Q}_{0}, \mathbf{Q}_{1} \ldots \mathbf{Q}_{N}\right\}^{T}
$$

The discretization of the boundary operator $\mathcal{I}$ as expressed in (20) is included in the right-hand side term $\mathbf{B}$ along with the terms from the other boundaries (gathered in $\mathbf{F}_{\mathbf{0}}$ but not detailed in this work):

$$
\mathbf{B}=\mathbf{F}_{\mathbf{0}}+\mathbf{F}_{\mathcal{I}}=\mathbf{F}_{\mathbf{0}}+\mathbf{F}-[\mathbf{C}] \mathbf{Q},
$$

where the $N_{F E}$ vector $\mathbf{F}$ and $N_{F E} \times 2(2 N+1)$ coupling matrix $[\mathbf{C}]$ come from the discretization of the first and second terms in (20), respectively. The evaluation of these terms must be handled with care since they might require integration of exponentialpolynomial products.

Likewise, the discretization of (22) yields the following relation:

$$
\left[\mathbf{C}^{\prime}\right] \mathbf{X}=\mathbf{F}^{\prime}-\left[\mathbf{A}^{\prime}\right] \mathbf{Q},
$$

where the $2(2 N+1) \times N_{F E}$ matrix $\left[\mathbf{C}^{\prime}\right]$, comes from the discretization of the left-hand-side term of (22) while the $2(2 N+1)$ vector $\mathbf{F}^{\prime}$ and the $2(2 N+1)$ diagonal matrix $\left[\mathbf{A}^{\prime}\right]$ come from the discretization of the first and second terms in the right-hand-side of (22). Note that $\mathbf{F}^{\prime}$ is zero for all $l$ except for $l=0$ 


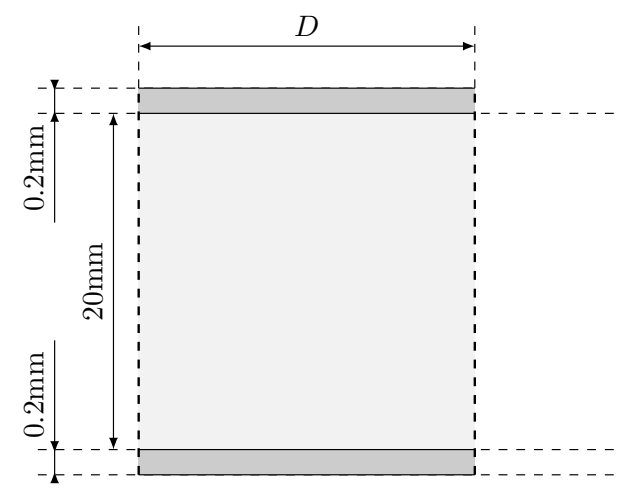

a)

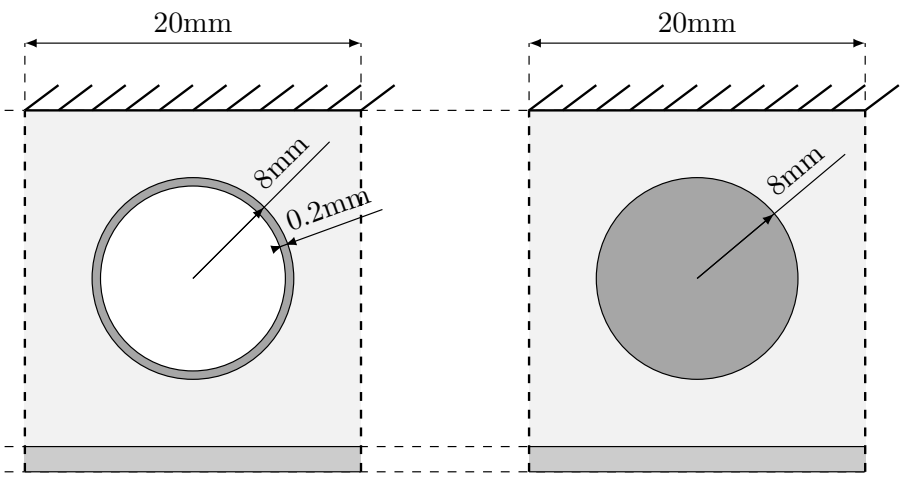

b) c)

Figure 2: The three elementary cells used in the examples, a) rubber-poroelastic-rubber sandwich panel with no inclusion, b) rubber coated poroelastic slab with an air-filled rubber shell inclusion, c) rubber coated poroelastic slab with a poroelastic inclusion.

where it takes the value $D$. Finally, combining equations (23) to (26), the following overall FEM/Bloch coupled-system is formed:

$$
\left[\begin{array}{cc}
{[\mathbf{A}]} & {[\mathbf{C}]} \\
{\left[\mathbf{C}^{\prime}\right]} & {\left[\mathbf{A}^{\prime}\right]}
\end{array}\right]\left\{\begin{array}{l}
\mathbf{X} \\
\mathbf{Q}
\end{array}\right\}=\left\{\begin{array}{l}
\mathbf{F} \\
\mathbf{F}^{\prime}
\end{array}\right\}+\left\{\begin{array}{c}
\mathbf{F}_{\mathbf{0}} \\
\mathbf{0}
\end{array}\right\}
$$

Hence, the vector of the FEM unknowns $\mathbf{X}$ is extended by the $2(2 N+1)$ vector of unknowns $\mathbf{Q}$ from the Bloch expansions.

\subsection{Extension to transmission prob- lems and multi-coating configura- tions}

The method has been presented for the reflection problem on a meta-poroelastic laminate with a rigid backing and a single coating above the metaporoelastic layer. It is now extended to the transmis-

Extension to transmission problems The fields transmitted through the system are accounted for in a manner similar to the reflected ones. Based on Bloch wave expansion, expressions similar to (2) can be written for the transmitted fields. This implies introducing a new set of unknown coefficients $\mathbf{Q}_{\mathbf{T} l}$ to be added to the linear system. The continuity relations between the FE domain and the coatings are rewritten in terms similar to (17). This procedure leads to a new exten-

$$
\left[\begin{array}{ccc}
{[\mathbf{A}]} & {[\mathbf{C}]} & {\left[\mathbf{C}_{\mathbf{T}}\right]} \\
{\left[\mathbf{C}^{\prime}\right]} & {\left[\mathbf{A}^{\prime}\right]} & {[\mathbf{0}]} \\
{\left[\mathbf{C}_{\mathbf{T}}^{\prime}\right]} & {[\mathbf{0}]} & {\left[\mathbf{A}_{\mathbf{T}}^{\prime}\right]}
\end{array}\right]\left\{\begin{array}{c}
\mathbf{X} \\
\mathbf{Q} \\
\mathbf{Q}_{\mathbf{T}}
\end{array}\right\}=\left\{\begin{array}{c}
\mathbf{F} \\
\mathbf{F}^{\prime} \\
\mathbf{0}
\end{array}\right\}
$$

with $\left[\mathbf{A}^{\prime}\right],\left[\mathbf{C}^{\prime}\right],[\mathbf{C}], \mathbf{Q}, \mathbf{F}^{\prime}$ being related to the reflection side and their counterparts subscripted with $\mathbf{T}$ to the transmission one. Note that the second forcing vector is null since no excitation is considered on the transmission side.

Extension to multi-coating configurations For multi-coating configurations, the approach relies on the modal Transfer Matrix Method (TMM) to transfer the fields through the different coating layers. A generalised transfer matrix for the whole multilayer can be deduced by multiplying the transfer matrices $[\mathbf{T}(n)]$ related to each of the $N_{L}$ layers in the system $[20]$. This leads to a new set of $\left[\mathbf{T}_{l}\right]$ matrices that can be substituted into (14) to account for a multilayer coating.

\section{Applications}

Three different cases are presented in this section, one is a transmission case without inclusion and the two others are reflection cases with periodic embeddings. The reference for the transmission case is computed using the TMM. On the other hand, the reflection cases reference solutions are computed using a semi-analytical Multiple Scattering [21] approach. Schematics of the test cases can be found in figure 2 and physical properties of the materials in table 1 .

\subsection{Transmission Case}

This first test computes the absorption coefficient and transmission loss of a rubber-poroelastic-rubber sandwich panel. The problem is of infinite extent along the $x$ direction, which is enforced using periodicity conditions on both sides of an elementary cell depicted in figure 2(a). Two tests are conducted: one with a period $D=20 \mathrm{~mm}$ and another with $D=150 \mathrm{~mm}$ and both use 10 elements per period. The goal of the second case is to demonstrate that the proposed approach allows reducing the number of elements while 
Table 1: Physical parameters used in the test suite, taken from Ref. [21] or manufacturer data (inclusion's poroelastic material is based on a sample from Eurocell).

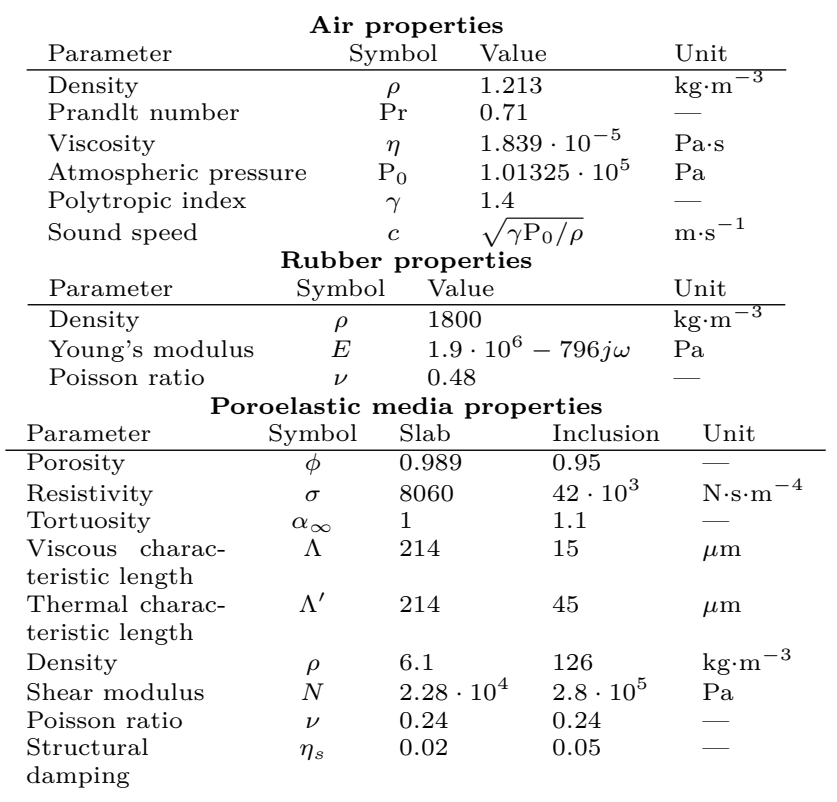
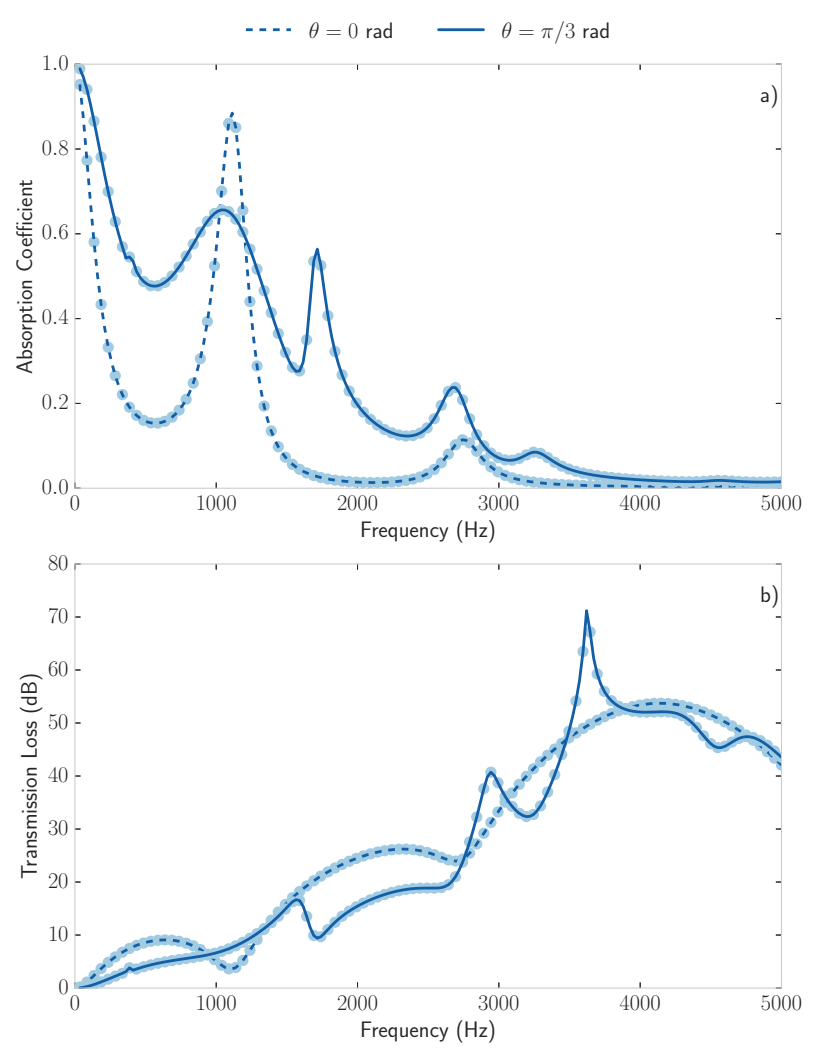

Figure 3: Absorption coefficient \& transmission loss of a rubber-poroelastic-rubber panel for two angles. The proposed method is plotted in solid line and the reference data with markers of similar shade.

controlling the loss of accuracy. A plane wave impinges the surface of the multilayer at an angle $\theta$ with respect to the normal to the surface and, given the problem geometry, only specular reflection and transmission might exist. In order to test the proposed method, the solution is computed considering 11 Bloch waves both in transmission and reflection and as many terms in each of the two expansions for $u_{x}$. The higher-order reflection and transmission coefficients are all expected to be null.

Using the TMM solution as a reference, one can validate the proposed approach by computing the evolution of the absorption coefficient and transmission loss with regard to the frequency. The results for this test are presented in figure 3 where solid lines correspond to the proposed method and the markers to the TMM reference. This figure shows a perfect agreement between both methods over the whole frequency range and particularly around the peaks of large absorption and transmission. The FE mesh is sufficiently refined and this first result suggests that the proposed method does not induce any loss of precision.

In order to check that the solution vector is consistent with the expectations, one may plot the evolution of the coefficients from the Bloch series with respect to the frequency, as proposed in figure 4. The nullorder coefficients (specular) are plotted in figure 4(a) and the higher-order ones in 4(b). One clearly sees that, except for the coefficients $R_{0}$ and $T_{0}$, all values are down to the order of $10^{-7}$. This validates that the method gives consistent results and does not generate artifacts.

In order to highlight another ability of the method, the period is increased $(D=150 \mathrm{~mm})$ while keeping the same number of elements. The comparison is made between the TMM reference (same as above), a solution using the proposed approach and a pure FEM solution modeling both the core and the coating using the FEM. Because of the period increase, the elements are slightly larger than in the previous case and the agreement with the reference solution is expected to be worse.

The results are shown in figure 5 with the TMM reference in black dots, the proposed method in light solid line and the FEM in dark solid line. One sees that at low frequency, all approaches produce similar results. As the frequency increases, the underrefined mesh leads to discrepancies in the pure FEM, the method being unable to represent the phenomena above $1 \mathrm{kHz}$. This is a side effect of distortion induced by an insufficiently refined mesh. The proposed approach does not experience the same issue: the coarse mesh tends to produce a slight shift of the peak towards low frequencies compared to TMM but the agreement is still good even without adding elements. Up to $5 \mathrm{kHz}$, no major disagreement between the TMM reference and the proposed approach can be noted, neither in amplitude nor peaks positions. 

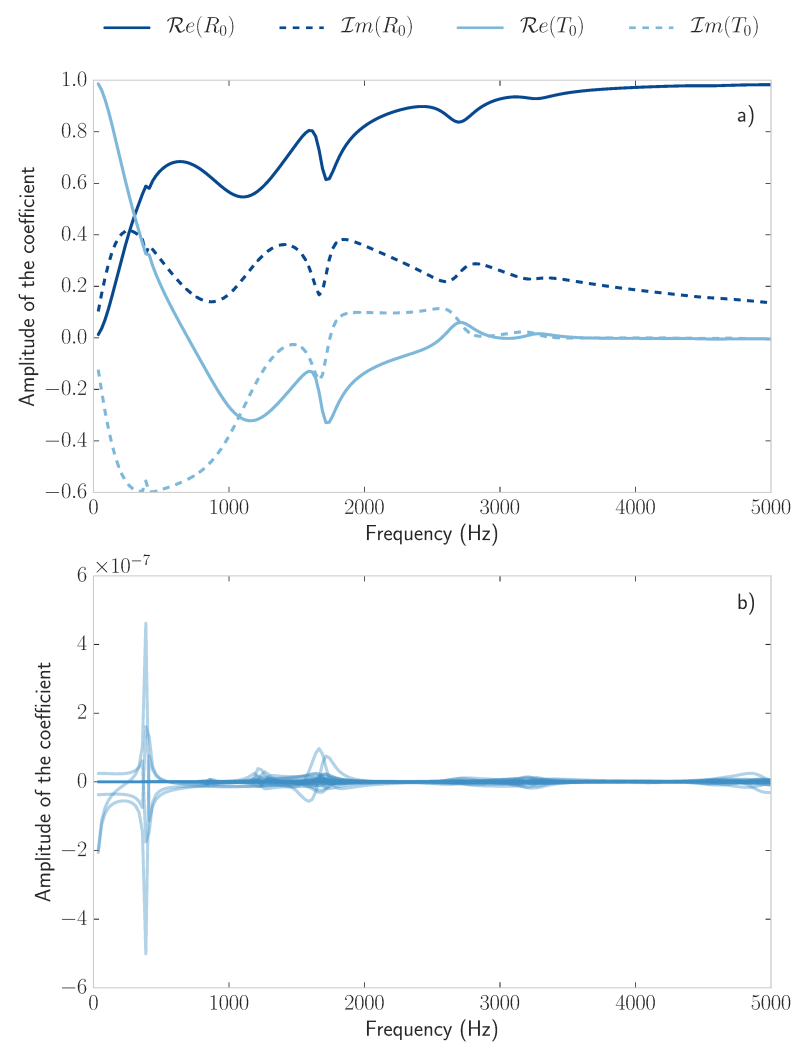

Figure 4: Bloch coefficients for a rubber-poroelasticrubber panel with and incidence angle $\theta=\pi / 3$. a) Null-order coefficients, real part in solid line and imaginary part in dashed line. b) Higher order coefficient with real and imaginary part superimposed (note the $10^{-7}$ scale factor).

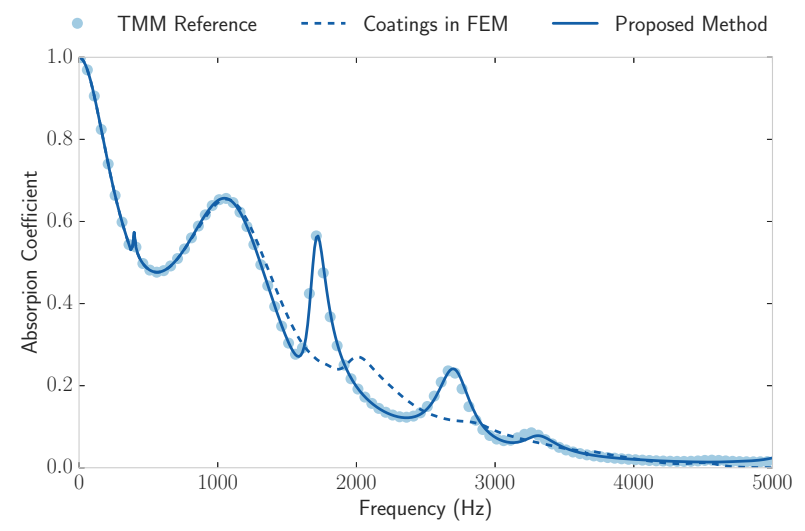

Figure 5: Comparison of the absorption coefficient computed using TMM (black dots), the proposed methods (light) or including the coatings in a Finite Element model. The number of elements in the core is the same for the two last methods, chosen such as one of them cat least complies with the reference.

\subsection{Reflection cases with inclusions}

To illustrate the effects of the meta-poroelastic laminates on wave reflection, two configurations are stud-
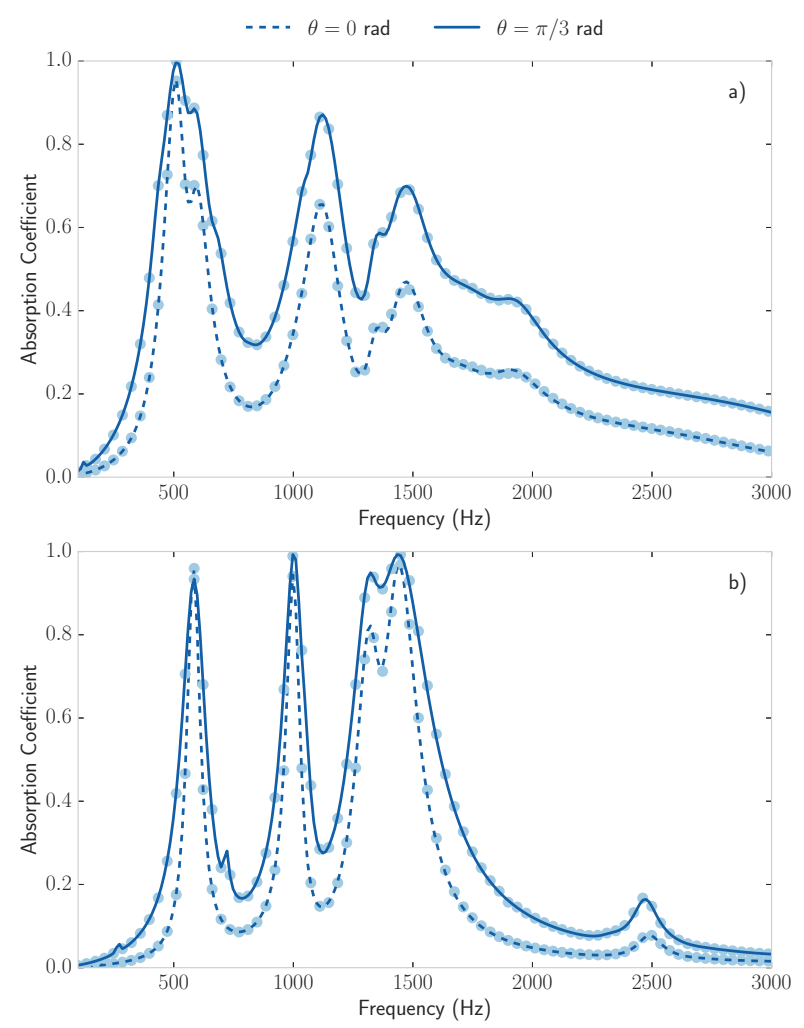

Figure 6: Absorption for two meta porous panels bonded on a rigid backing with air-filled rubber shell (top) or poroelastic (bottom) circular inclusion. The panels are coated with rubber and excited by a plane wave for two different incidence angles. multiple scattering results (disc markers) are plotted against those from the proposed method (solid line)

ied. In the first case, the inclusion is made of poroelastic material, while in the second case the inclusion consists of a rubber shell with a $0.2 \mathrm{~mm}$ thickness filled with air, see figures 2(b) and 2(c). In both configurations, circular inclusions have an external radius of $8 \mathrm{~mm}$, the period is $D=20 \mathrm{~mm}$, the poroelastic matrix is $20 \mathrm{~mm}$ thick and is placed on a rigid backing while its free surface is coated with a $0.2 \mathrm{~mm}$ rubber layer. Results are presented in figure 6, and are compared with those obtained by the Multiple Scattering Theory for poroelastic media [21]. Two angles of incidence are considered: normal incidence $(\theta=0)$ and oblique incidence with $\theta=\pi / 3$. The overall agreement between both methods is excellent and both the amplitude and the position of maxima match well.

\section{Conclusion}

In the present work, a method to account for thin plane structures at the boundary of a Finite-Element model is proposed. This method does not require to mesh the considered coating, limiting both the increase in complexity of the model and the potential 


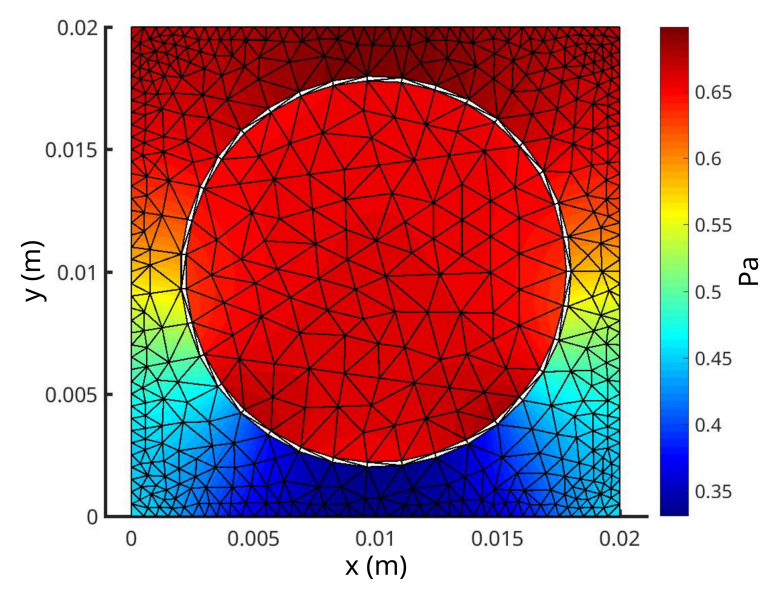

(a) Fluid pressure

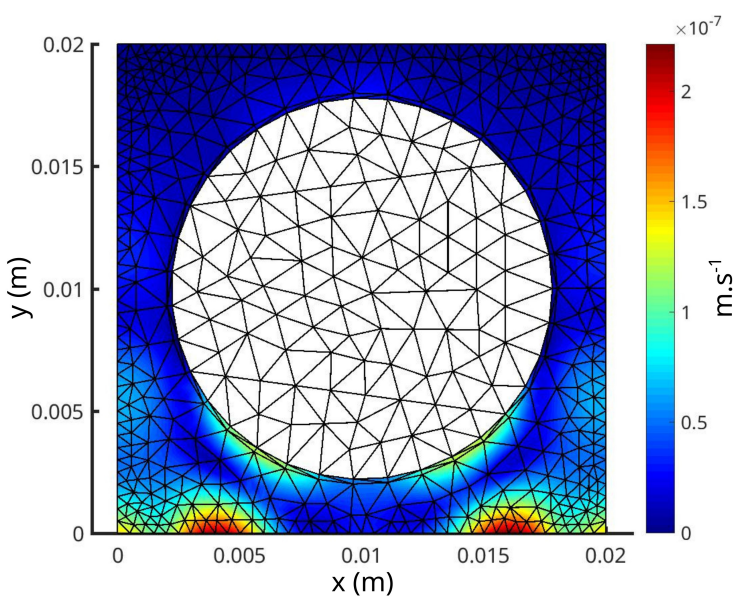

(b) Solid displacement

Figure 7: Fields' maps for the rubber shell case at $f=1945 \mathrm{~Hz}$ and $\theta=\pi / 6$.

distortion effects. The method is based on a transfer matrix approach and allows accounting for singleand multilayer coatings for a negligible increase of the model's size, while keeping the versatility of the FEM. The proposed approach was compared to the semianalytical Multiple Scattering Technique to assess its the method is thus deemed reliable for the considered cases. Although the theoretical discussion was focused on a single layer elastic coating placed on a meta poroelastic layer, the approach could be extended to ther types of media (anisotropic, for instance).

The whole development has been thought for meta materials design. Actually, the technique presented in this work has the potential to facilitate design of new meta absorbers. It alleviates the cost of meshing the coatings and allows to resolve separately all Bloch coefficients while still taking advantage of the FEM's versatility. Based on the FEM, the method allows to visually explore the on-going phenomena by generating maps as could be done with pure FEM typically implemented in commercial software. Examples of such maps for the system presented in figure 2(b) are depicted in figure 7 . These field representations allow to finely investigate localization and radiation properties of such systems and can eventually serve as a guide towards new designs.

Applications of this work may concern a numerical approach to the design of meta materials and meta poroelastic laminates, especially in industrial applications where multilayer coatings are heavily used for protection, aesthetics, etc. and where the efficient simulation of their effect is crucial to the design process.

\section{Acknowledgements}

O. D., L. S., and J.-P. G. are grateful to the French ANR project Metaudible (ANR-13-BS09-0003). This article is based upon work from COST Action DENORMS CA15125, supported by COST (European Cooperation in Science and Technology).

\section{References}

[1] Logan Schwan, Olga Umnova, and Claude Boutin. Sound absorption and reflection from a resonant metasurface: Homogenisation model with experimental validation. Wave Motion, 72:154-172, July 2017.

[2] Matthew D. Guild, Victor M. García-Chocano, Weiwei Kan, and José Sánchez-Dehesa. Acoustic metamaterial absorbers based on multilayered sonic crystals. Journal of Applied Physics, 117(11):114902, March 2015.

[3] J.-P. Groby, C. Lagarrigue, B. Brouard, O. Dazel, V. Tournat, and B. Nennig. Enhancing the absorption properties of acoustic porous plates by periodically embedding Helmholtz resonators. The Journal of the Acoustical Society of America, 137(1):273-280, 2015.

[4] C. Lagarrigue, J. P. Groby, V. Tournat, O. Dazel, and O. Umnova. Absorption of sound by porous layers with embedded periodic arrays of resonant inclusions. The Journal of the Acoustical Society of America, 134(6):4670-4680, 2013.

[5] J. V. Sánchez-Pérez, D. Caballero, R. MartinezSala, C. Rubio, J. Sánchez-Dehesa, F. Meseguer, J. Llinares, and F. Gálvez. Sound attenuation by a two-dimensional array of rigid cylinders. Physical Review Letters, 80(24):5325, 1998.

[6] T. Weisser, J.-P. Groby, O. Dazel, and L. Schwan. High broadband absorption of acoustic waves by elastic-framed metaporous layer. In Advanced Electromagnetic Materials in Microwaves and $\mathrm{Op}$ tics (METAMATERIALS), 2016 10th International Congress on, pages 322-324. IEEE, 2016.

[7] Claude Boutin and Pascale Royer. On models of double porosity poroelastic media. Geophysical Journal International, 203(3):1694-1725, 2015. 
[8] Cesar R. Garcia, Jesus Correa, David Espalin, Jay H. Barton, Raymond C. Rumpf, Ryan Wicker, and Virgilio Gonzalez. 3d printing of anisotropic metamaterials. Progress In Electromagnetics Research Letters, 34:75-82, 2012.

[9] Noé Jiménez, Vicent Romero-Garcia, Vincent Pagneux, and Jean-Philippe Groby. Quasiperfect absorption by subwavelength acoustic panels in transmission using accumulation of resonances due to slow sound. Physical Review B, 95(1), 2017.

[10] Muamer Kadic, Tiemo Bückmann, Nicolas Stenger, Michael Thiel, and Martin Wegener. On the practicability of pentamode mechanical metamaterials. $A p$ plied Physics Letters, 100(19):191901, 2012.

[11] C. Lagarrigue, J.-P. Groby, O. Dazel, and V. Tournat. Design of metaporous supercells by genetic algorithm for absorption optimization on a wide frequency band. Applied Acoustics, 102:49-54, 2016.

[12] Panos M. Pardalos, Piero Conca, Giovanni Giuffrida, and Giuseppe Nicosia, editors. Machine Learning, Optimization, and Big Data, volume 10122 of Lecture Notes in Computer Science. Springer International Publishing, Cham, 2016. DOI: 10.1007/978-3-31951469-7.

[13] L. C. Botten, R. C. McPhedran, N. A. Nicorovici, A. A. Asatryan, C. M. De Sterke, P. A. Robinson, K. Busch, G. H. Smith, and T. N. Langtry. Rayleigh multipole methods for photonic crystal calculations. Progress In Electromagnetics Research, 41:21-60, 2003.

[14] O. C. Zienkiewicz and Robert L. Taylor. The finite element method: the basis. Butterworth-Heinemann, Oxford ; Boston, 5th ed edition, 2000.

[15] Noureddine Atalla, Raymond Panneton, and Patricia Debergue. A mixed displacement-pressure formulation for poroelastic materials. The Journal of the Acoustical Society of America, 104(3):1444, 1998.

[16] Noureddine Atalla, M. A. Hamdi, and Raymond Panneton. Enhanced weak integral formulation for the mixed (u,p) poroelastic equations. The Journal of the Acoustical Society of America, 109(6):3065, 2001.

[17] H. Bufler. Theory of elasticity of a multilayered medium. Journal of Elasticity, 1(2):125-143, 1971.

[18] J.-P. Groby, Armand Wirgin, and Erick Ogam. Acoustic response of a periodic distribution of macroscopic inclusions within a rigid frame porous plate. Waves in Random and Complex Media, 18(3):409433, 2008.

[19] J.-P. Groby, W. Lauriks, and T. E. Vigran. Total absorption peak by use of a rigid frame porous layer backed by a rigid multi-irregularities grating. The Journal of the Acoustical Society of America, 127(5):2865-2874, 2010.

[20] J.-F. Allard and Noureddine Atalla. Propagation of sound in porous media: modelling sound absorbing materials. Wiley, Hoboken, N.J, 2nd ed edition, 2009.

[21] Thomas Weisser, Jean-Philippe Groby, Olivier Dazel, François Gaultier, Elke Deckers, Sideto Futatsugi, and Luciana Monteiro. Acoustic behavior of a rigidly backed poroelastic layer with periodic resonant inclusions by a multiple scattering approach. The Journal of the Acoustical Society of America, 139(2):617-629, 2016 . 